\title{
AUTOEVALUACIÓN DE ELEMENTOS DE LA NORMA ISO 9001:2015 EN UN GRUPO DE MIPYMES COSTARRICENSES
}

\author{
ING. JEINER MORA ABARCA \\ Universidad Fidélitas, Costa Rica \\ jemora@ufidelitas.onmicrosoft.com \\ ING. RUTH RODRÍGUEZ VILLALOBOS \\ Universidad Fidélitas, Costa Rica \\ ruth.rodriguezv@ufide.ac.cr
}

\section{RESUMEN}

Las micro, pequeñas y medianas empresas costarricenses cumplen un papel significativo en el crecimiento económico del país. Proveer productos y servicios de calidad de manera sostenida resulta ineludible; sin embargo, la práctica de algún sistema que ayude a controlar su calidad suele ser una tarea difícil. La Universidad Fidélitas y la Cámara de Exportadores de Costa Rica realizaron una encuesta de auto-calificación entre julio y octubre de 2018 a un grupo de empresas de este tipo para conocer cómo gestionan su calidad (con referencia a la Norma ISO 9001:2015) considerando variables como: tamaño de la empresa, sector y condición de exportación. Los resultados demuestran que la principal variable es el tamaño de la empresa; ya que, conforme aumenta el número de empleados, se aplican más robustamente los principios de la Norma. Las secciones de la Norma que implican actividades de ejecución y seguimiento fueron evaluadas con un nivel más bajo.

PALABRAS CLAVE: MICRO, PEQUEÑAS Y MEDIANAS EMPRESAS, CALIDAD, GESTIÓN DE CALIDAD.
ABSTRACT

Micro, small and medium-sized Costa Rican companies play a significant role in the economic growth of the country. Providing quality products and services in a sustained manner is unavoidable, however, the practice of an organized system that helps them to control its quality is usually a difficult task. Universidad Fidélitas and the Chamber of Exporters of Costa Rica, conducted a self-rating survey from July to October 2018 to a group of companies of this type to know how they manage their quality (with reference to ISO 9001: 2015) considering variables as: size of the company, sector and condition of export. The results show that the main variable is the size of the company, since as the number of employees increases, the principles of the Standard are more robustly applied. Sections of the Standard that imply execution and monitoring activities were evaluated at a lower level.

KEYWORDS: MICRO, SMALL AND MEDIUM-SIZED ENTERPRISES, QUALITY, QUALITY MANAGEMENT SYSTEMS. 


\section{INTRODUCCIÓN}

Las pequeñas y medianas empresas (MIPYMES) costarricenses cumplen un papel fundamental en el crecimiento económico del país por medio de la generación de empleo y contribución a las exportaciones. Casi el 15\% de las exportaciones totales en Costa Rica, en el año 2016, corresponden a MIPYMES (Cámara de Exportadores de Costa Rica, CADEXCO, 2017). Esto es relevante considerando el volumen en exportaciones que representan las compañías multinacionales. Sin embargo, la contribución de las MIPYMES al total de las exportaciones bajó en un 1.17\% del 2015 al 2016 (Ministerio de Economía, Industria y Comercio, MEIC, 2017).

Para cumplir con los requisitos de los mercados internacionales, las empresas exportadoras deben, entre otros factores, demostrar su capacidad empresarial. Uno de los elementos más importantes es alcanzar niveles de calidad de manera sostenida, los cuales se logran, en la mayoría de los casos, con un sistema de gestión de calidad y normas o certificaciones internacionales.

Existe, por lo tanto, un interés por evaluar el quehacer de las MIPYMES que actualmente mantienen procesos de exportación o han mostrado interés en exportar sus productos. Este conjunto de empresas constituye una fuente de información importante para entender sus fortalezas o debilidades en el campo de la gestión de calidad. Dado que la familia de normas ISO 9001 es una de las más completas y conocidas en la industria, esta se propone como base para el presente estudio para evaluar los principios del sistema de gestión de calidad.

La Cámara de Exportadores de Costa Rica (CADEXCO), ha iniciado un proyecto cuyo fin es capacitar y ayudar a los empresarios de MIPYMES en relación con los requisitos para exportación. La Universidad Fidélitas y CADEXCO han acordado un convenio para conocer, por medio de esta investigación, el quehacer de las MIPYMES con respecto al tema de la calidad, ya que este es uno de los aspectos más importantes para la selección de las empresas que van a ser sujeto de atención en este proyecto.

El decrecimiento en el volumen de exportaciones de las PYME entre el 2015 y 2016 enciende una alerta en relación con las posibles causas del fenómeno. Sin duda, la calidad y su gestión juegan un papel de vital importancia en el crecimiento, sostenibilidad y capacidad de exportación de las empresas. Surge, por lo tanto, la siguiente pregunta de investigación: ¿cómo se gestiona la calidad en un grupo de MIPYMES costarricenses, considerando variables como tamaño, sector y condición de exportación?

El objetivo general este estudio es realizar una autoevaluación del grado de cumplimiento de los principales elementos de un sistema de gestión de calidad, basado en la Norma ISO 9001:2015, entre un grupo de MIPYMES costarricenses afiliadas a CADEXCO. Lo anterior se logra mediante el desarrollo de un instrumento de recolección de datos para determinar el grado de cumplimiento de los principales elementos de un sistema de gestión de calidad (basado en la Norma ISO 9001:2015). Luego, se realiza una comparación entre el grado de cumplimiento de los elementos del sistema de gestión, el sector al que pertenece la MIPYME, el tamaño de la empresa y su condición de exportación.

\section{MICRO, PEQUEÑAS Y MEDIANAS EMPRESAS (MIPYMES)}

Una pequeña y mediana empresa (PYME) es "toda unidad productiva de carácter permanente que disponga de los recursos humanos, los maneje y opere, bajo las figuras de persona física o de persona jurídica, en actividades industriales, comerciales, de servicios o agropecuarias que desarrollen actividades de agricultura orgánica" (MEIC, s.f.). El término MIPYME surge al incorporar a la micro empresa (Instituto Nacional 
de Aprendizaje, INA, 2018). Para definir el tamaño de una empresa, una de las clasificaciones más comunes en nuestro país es la del MEIC, la cual indica que:

El tamaño se determina mediante la ponderación matemática de una fórmula que las clasifica según la actividad empresarial, y que contempla el personal promedio contratado en un período fiscal, el valor de los activos, el valor de las ventas anuales netas y el valor de los activos totales netos (INA, 2018).

Para efectos de esta investigación, no se toma en cuenta el aspecto financiero de la empresa. Se considera micro empresa si el número de empleados es igual o menor a 10; pequeña empresa entre 11 y 35 empleados y, entre 36 y 100 empleados, se considera una empresa mediana.

En el 2016 solamente 6197 MIPYMES, de un total de 21179, estaban activas en el Sistema de Información Empresarial Costarricense (SIEC). Del 2015 al 2016 se dio una reducción de un 9.6\% de MIPYMES activas. Es decir, desde el 2011 hay un crecimiento importante de empresas MIPYMES registradas; sin embargo, el número de empresas inactivas ha tenido un considerable aumento. Esto refleja que un buen número de empresarios inician con su empresa, pero después de un tiempo se vuelven inactivas.

\section{MIPYMES, CALIDAD Y EXPORTACIONES}

Sobre las MIPYMES, Arce, Méndez y Villegas (2017) indican que "en muchos casos, los dueños de las MIPYMES realizan una gestión empírica con base en su experiencia y no conocimiento técnico y especializado, lo que genera que en algunas ocasiones no sean administradas adecuadamente" (p. 2). Esta debilidad implica una dificultad para implementar sistemas de gestión de calidad y, por lo tanto, organizar la empresa para dar un salto al exterior y lograr que la empresa sea competente para realizar exportaciones.
Por otro lado, las MIPYMES muchas veces son empresas de pocos ingresos, escasa posibilidad de inversión, una estructura organizacional muy simple y con poco tiempo para tareas de planificación. Estas condiciones podrían incidir en la habilidad de gestionar la calidad y de realizar exportaciones. La calidad y la mejora continua, aspectos que se pretende alcanzar de manera sostenida por medio de los sistemas de gestión de calidad, son elementos fundamentales para las empresas que pretenden abrir sus mercados al extranjero.

Las empresas que hoy en día deseen exportar "deben buscar la excelencia y la calidad en sus productos y servicios, siempre pensando en mercados internacionales donde la exigencia es mayor" (Chacón, 2016). Por tanto, es importante realizar una valoración para determinar la relación existente entre sistemas de calidad y las exportaciones de las MIPYMES.

Alrededor del mundo se han realizado numerosos estudios sobre el quehacer de las MIPYMES con respecto a la gestión de la calidad de sus productos o servicios, y la importancia de la aplicación de sistemas de control organizados. Israr y Gangele (2014), en un estudio realizado a pequeñas empresas en la India, encuentran que en general estas no trabajan con un compromiso hacia la calidad. La escogencia de los suplidores de materias primas generalmente se basa solamente en el precio del producto (descuidando la calidad) y no organizan programas de entrenamiento para sus trabajadores. Encuentran también en su estudio, que el liderazgo administrativo y la participación de los trabajadores son aspectos fundamentales que se necesitan para que los programas de control de calidad tengan éxito.

Por ejemplo, en un estudio realizado en España en 1997 con 53 empresas en Cádiz, se encontró que la Gestión de Calidad en micro, pequeñas y medianas empresas tiene enfoque estratégico, por lo que la deficiente gestión de calidad frena 
su desarrollo. Nuevamente en España, en el año 2009, Rubio y Aragón realizaron un estudio a 1201 MIPYMES en el que demostraron que uno de los factores de éxito es la calidad. Sin embargo, solo el 20\% contaba con una certificación de ISO 9000 o similar, con lo que se demostraba que las empresas de menor tamaño expresaban poco interés de adaptarse a las exigencias de los mercados actuales.

En México se realizó, en el 2014, un estudio de 400 MIPYMES, en donde se determinó que el $71 \%$ muestra baja y muy baja competitividad en el área de Aseguramiento de la Calidad. Solo el $52 \%$ se ha preocupado por contar con una filosofía de Calidad y el 62\% ha implementado Normas de Calidad UNAM (2018).

En la publicación de Rodríguez, Bañuelos y Chávez (2010), se realizó una investigación para determinar los factores internos que afectan a la competitividad de las PYME en el sur de Tamaulipas, México. Tomaron una muestra de 100 empresas y los factores internos de mayor impacto son: la planeación estratégica, las habilidades directivas del responsable o gerente de la empresa, la calidad del servicio y la tecnología de información y comunicación.

Con base en la información anterior, las organizaciones deben considerar aspectos clave como la capacitación del personal, mejora continua de procesos e incorporación de elementos de control de calidad. Lo anterior para mejorar este último aspecto en el servicio, además de la satisfacción de los clientes, el éxito competitivo que se requiere $y$, por lo tanto, aumentar las posibilidades de exportación de sus productos.

Hoy en día, las industrias deben preocuparse por brindar servicios y productos de calidad, inclusive en los congresos internacionales se enmarca como uno de los temas críticos a revisar. Por ejemplo, en el 2016, en México, se llevó a cabo el XXI Congreso Internacional de Contaduría, Administración e Informática y en él se analizaron una serie de temas relacionados con las MIPYMES, en donde se destacó el tema de la calidad para la competitividad.

En este congreso se trataron temas como el marco de medición para las MIPYMES, el cual se desarrolló por parte del Banco Interamericano de Desarrollo (BID), llamado el Mapa de Competitividad y se adaptó por parte de la Cámara de Comercio de Medellín y Antioquía, ello para utilizarse como un diagnóstico organizacional de las MIPYMES en Colombia. El mapa de la competitividad se muestra en la tabla 1.

En este mapa de competitividad, el cual es basado en el documento del BID, se destaca el apartado de Aseguramiento de la Calidad, el cual debe ser objeto de estudio para las MIPYMES. Se puede ver cómo el tema de Calidad es un elemento que no se puede dejar de lado si se quiere tener éxito empresarial. Sin embargo, los resultados de los diferentes estudios reflejan que, comúnmente, las empresas que están en la categoría de MIPYMES no tienen como una de sus prioridades los elementos de calidad para ser competitivas.

Por otro lado, la calidad para la competitividad se ha vuelto primordial, ya que el mercado es cada vez más exigente. Rubio y Aragón (2009) indican lo que

Estudios muestran el impacto positivo de la calidad sobre la productividad, la exportación, el control de la empresa, los costos, la reducción de las quejas de los clientes y la imagen de la empresa, variables todas ellas ligadas directamente a la competitividad (p.193).

En un estudio realizado por Córdoba y Henderson (2017), en 165 MIPYMES de la región Huetar Caribe Costarricense, se obtiene como principal resultado que existe una correlación alta y positiva entre la gestión de calidad y el desempeño exportador. Esto significa que, a mayor desarrollo en sistemas de gestión de calidad, las empresas presentan un alto desempeño exportador. Los aspectos considerados en gestión de calidad 


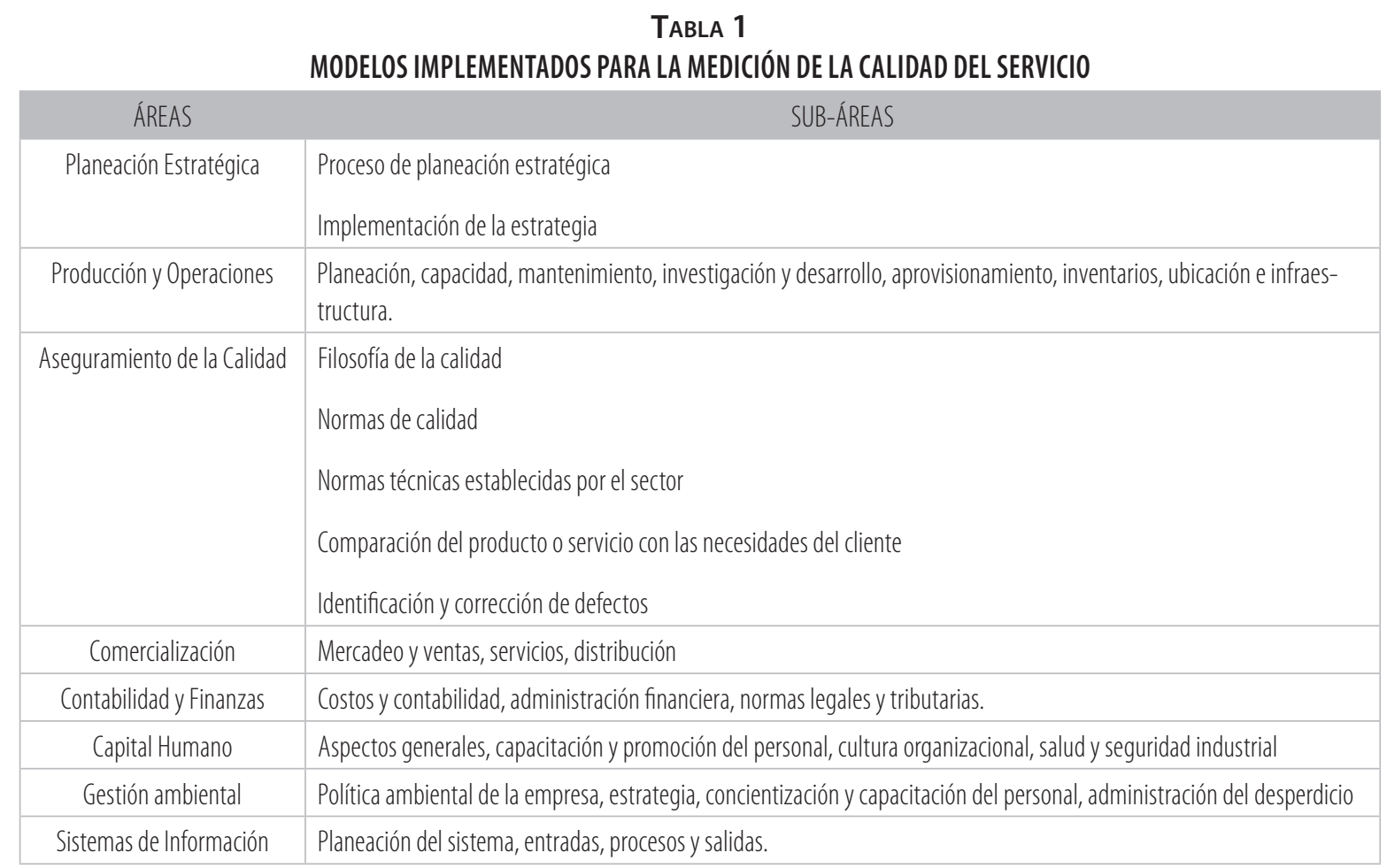

Fuente: UNAM (2018)

comprenden control de procesos, cultura hacia la calidad, uso de herramientas de mejora y existencia de normas de calidad. En lo relativo al desempeño exportador, se incluye continuidad, crecimiento y diversificación de mercados.

En Costa Rica, INTECO (Instituto de Normas Técnicas de Costa Rica), establece la Norma INTE 01-01-09:2013 especialmente para servir de guía a los empresarios de las MIPYMES en la gestión de sus procesos. Esta Norma integra sistemas de gestión de calidad y ambiente. Por medio de un proceso de mejora continua, facilita a las MIPYMES la implementación de otras normas internacionales.

\section{GESTIÓN DE CALIDAD Y NORMA ISO 9001:2015}

La Gestión de Calidad es una herramienta hoy en día utilizada por muchas empresas para ofrecer productos y procesos de calidad, así como también servicios. Una de las definiciones de Gestión de Calidad dictada por la Universidad Cooperativa de Colombia en su web

Una herramienta que le permite a cualquier organización planear, ejecutar y controlar las actividades necesarias para el desarrollo de la misión, a través de la prestación de servicios con altos estándares de calidad, los cuales son medidos a través de los indicadores de satisfacción de los usuarios (Universidad Cooperativa de Colombia, UCC, 2018).

Con el fin de elaborar normas para la Gestión de la Calidad en las empresas, la serie de normas ISO 9000 desarrolló estándares para la certificación de sistemas de gestión de calidad. El ISO (International Organization for Standardization) es el mayor desarrollador de estándares de calidad del mundo. Según la Norma ISO 9001:2015 Conceptos y Definiciones 
Un Sistema de Gestión de Calidad comprende actividades mediante las que la organización identifica sus objetivos y determina los procesos y recursos requeridos para lograr los resultados deseados. El Sistema de Gestión de Calidad gestiona los procesos que interactúan y los recursos que se requieren para proporcionar valor y lograr los resultados para las partes interesadas pertinentes (ISO 9001, 2015).

La nueva Norma ISO 9001:2015 también incluyó, dentro de su actualización, la gestión de riesgos, los cuales pueden convertirse en Oportunidades o Amenazas, un aspecto muy valioso para lograr competitividad en cada organización.

La Gestión de Riesgos está constituida por "las actividades coordinadas para dirigir y controlar la organización con relación al riesgo" ISO 31000 (2015). Un riesgo no solo es una amenaza, también puede ser una oportunidad. Las empresas que deseen exportar están expuestas a todo tipo de riesgo y basarse en la Norma ISO 9001:2015, les ayuda a gestionar oportuna y adecuadamente estos riesgos. Las secciones (de la 4 a la 10) en las que se basa la encuesta a aplicar a las MIPYMES son tomadas de la Norma ISO 9001:2015. Estas son:

Sección 4. Contexto de la Organización. La claridad en los aspectos tanto internos como externos para brindar productos y/o servicios de calidad. Deben definirse con anticipación.

Sección 5. Liderazgo. Desde la alta dirección debe haber un claro objetivo y compromiso con el sistema de gestión de calidad.

Sección 6. Planificación. Iniciando con el primer ítem de Mejora continua, la planificación es parte importante para una empresa que desea exportar sus productos o servicios. Toma en cuenta las acciones para abordar los riesgos y las oportunidades. Definición de la política, objetivos y la planificación para lograrlos.
Sección 7. Apoyo. La definición clara de los recursos, personas e infraestructura para el establecimiento, mantenimiento y mejora continua del sistema de gestión de calidad. Además, el ambiente apropiado para la realización de procesos.

Sección 8. Operación. En este elemento entra la planificación y el control operacional para que los procesos cumplan con los requisitos para la provisión de productos y servicios. Dentro de ellos está la comunicación con el cliente, determinación de requisitos del producto y servicios. Además, incluye el diseño y desarrollo de productos y servicios.

Sección 9. Evaluación del Desempeño. "La organización define los métodos de seguimiento, medición, análisis y evaluación necesarios para asegurar los resultados válidos" (NORMA ISO 9001:2015). Con ello, se asegura la evaluación de la eficiencia y eficacia de sus procesos, parte importante de un Sistema de Gestión de Calidad. Por otro lado, también realiza auditorías internas, las cuales documenta y da seguimiento, así como la revisión que realiza la Dirección de sus sistemas de calidad.

Sección 10. Mejora. Las oportunidades de mejora deben determinarse por parte de la organización y principalmente tomar acciones para cumplir con los requisitos del cliente, cuyo objetivo es aumentar su satisfacción. El buen tratamiento de los productos no conformes y de las no conformidades dadas en la prestación del servicio. Además, esta sección se trata de que toda la organización se enfoque en la mejora continua.

Es importante destacar que, como referencia, se toma la NORMA ISO 9001:2015 por ser un estándar mundial. En la versión 2015, la Norma presenta una comparación entre sus secciones y las fases del ciclo de mejora continua Planificar-HacerVerificar-Actuar (PHVA). Las Secciones 4 a 10 de la Norma están representadas en el Ciclo PHVA de mejora continua. Por ejemplo, la etapa Planificar está representada por las Secciones 4, 5 y 6 de la 
Norma: Contexto de la Organización, Liderazgo y Planificación. La etapa Hacer, está representada en las Secciones 7 y 8 de la Norma: Apoyo y Operación. Por otro lado, la etapa Verificar, está representada en la Sección 9 de la Norma: Evaluación del Desempeño. La etapa Actuar, está representada en la Sección 10 de la Norma: Mejora.

\section{METODOLOGÍA}

El estudio que aquí se presenta tiene un enfoque cuantitativo, con un alcance descriptivo. Mediante herramientas de recolección de información fue posible obtener datos numéricos concretos que sirvieron para describir, representar y caracterizar un fenómeno específico. Con el propósito de conocer la situación general en aspectos de calidad en un grupo de MIPYMES costarricenses, se confeccionó un cuestionario de 28 preguntas que fueron enviadas vía correo electrónico a los pequeños empresarios.

El estudio se realizó en convenio con CADEXCO. Esta institución tiene alrededor de 300 empresas afiliadas; por tanto, la población se considera: $\mathrm{N}=$ 300. Por medio de un muestreo no aleatorio, por convenio, CADEXCO proporcionó la información de contacto de 200 empresas ( $N=200$ ) a las cuales se les envió la encuesta. Estas empresas están todas en la categoría MIPYMES. El cuestionario fue respondido por 77 empresarios.

Las variables que se consideraron en este estudio para las MIPYMES son:

- Condición de exportación

- Sí exporta

- No exporta

- Tiene intención de exportar

- Tamaño de la empresa

- Micro empresa
- Pequeña empresa

- Mediana empresa

- Tipo de empresa

- Manufactura

- Servicios

- Ambas

Se utilizó un cuestionario de 28 preguntas que consta de dos partes. La primera parte está conformada por un grupo de 8 preguntas abiertas y de selección, donde se consultó, además del número de empleados, a qué se dedica la empresa, si exportan o tienen expectativas de exportar, cuáles son sus principales problemas en relación con la calidad, cuál es el sistema de calidad que utilizan actualmente, entre otros. La segunda parte está conformada por 20 preguntas sobre algunos de los aspectos más relevantes de la Sección 4 a la Sección 10 de la Norma ISO 9001:2015. La evaluación se realizó mediante una modificación de la escala Likert. Se usaron las alternativas: no aplica, en desacuerdo, poco de acuerdo, de acuerdo y muy de acuerdo.

En la tabla 2 se presenta un resumen de las preguntas realizadas en la segunda parte del cuestionario, según sección de la Norma ISO 9001:2015 a la que corresponden.

Se utilizó un lenguaje sencillo para que los pequeños empresarios puedan autoevaluar su situación en cada una de las secciones. Las preguntas se hicieron con referencia a aspectos generales de control de calidad y no a una norma en sí, debido a que no necesariamente deben estar certificados en un Sistema de Gestión de Calidad o conocer su significado para poder responder el cuestionario. El cuestionario es original de los autores.

Con el fin de determinar la fiabilidad del instrumento de recolección de datos, se realiza un análisis a las preguntas evaluadas mediante la escala 
TABLA 2

RESUMEN DE LAS PREGUNTAS DE LA ENCUESTA

\begin{tabular}{|c|c|}
\hline SECCIÓN DE LA NORMA & PREGUNTA \\
\hline \multirow[t]{3}{*}{ 4. Contexto de la Organización } & ¿Tiene algún tipo de Sistema de Control de Calidad? \\
\hline & ¿Conoce y satisface las necesidades de sus clientes? \\
\hline & ¿Los procesos están documentados? \\
\hline \multirow[t]{3}{*}{ 5. Liderazgo } & ¿Tiene una Política de Calidad? \\
\hline & ¿La Política es comunicada a todo el personal? \\
\hline & ¿Se hacen esfuerzos por aumentar satisfacción de sus clientes? \\
\hline \multirow[t]{3}{*}{ 6. Planificación } & ¿Empleados perciben compromiso de la Gerencia hacia la calidad? \\
\hline & ¿La empresa tiene definidos objetivos de calidad? \\
\hline & ¿Los objetivos se pueden medir, controlar y hay un responsable? \\
\hline \multirow[t]{3}{*}{ 7. Apoyo } & ¿El personal de Control de Calidad tiene formación y experiencia? \\
\hline & ¿Está documentado el Sistema de Control de Calidad? \\
\hline & ¿Se comunica a los empleados asuntos del Sistema de Control de Calidad? \\
\hline \multirow[t]{4}{*}{ 8. Operación } & ¿Existe un sistema organizado para atender las quejas de los clientes? \\
\hline & ¿Existe un sistema para verificar que se cumpla con lo ofrecido al cliente? \\
\hline & ¿Existe un control de especificaciones para materias primas? \\
\hline & ¿El sistema evita que el producto no conforme le llegue al cliente? \\
\hline \multirow[t]{2}{*}{ 9. Evaluación del Desempeño } & ¿Realizan auditorías internas para verificar Sistema de Control de Calidad? \\
\hline & ¿Hay algún mecanismo de seguimiento al grado de satisfacción del cliente? \\
\hline \multirow[t]{2}{*}{ 10. Mejora } & ¿Se ejecutan acciones para controlar y corregir no conformidades? \\
\hline & ¿Se conserva documentación relativa a las no conformidades? \\
\hline
\end{tabular}

Fuente: Elaboración propia

Likert, utilizando el Coeficiente Alfa de Cronbach. Este coeficiente mide la fiabilidad en función de dos términos: el número de ítems (o longitud de la prueba) y la proporción de varianza total de las respuestas de la prueba. El análisis se realizó mediante el Software SPSS. El resultado obtenido es de 972 para el Coeficiente Alfa de Cronbach, y con este dato se considera excelente la consistencia interna del instrumento. Por otro lado, el porcentaje de error para la muestra es de un $11 \%$.

La base de datos de las MIPYMES fue proporcionada por CADEXCO, en el marco de un estudio preliminar para un proyecto especial de apoyo a los empresarios. Se enviaron 200 cuestionarios vía correo electrónico utilizando la herramienta Google Forms. Las respuestas al cuestionario fueron analizadas mediante el uso del software SSPS versión 24.

\section{RESULTADOS}

Un 55\% de las empresas encuestadas pertenece al sector servicios; un 36\% al sector manufactura y un 9\% se dedica a ambas actividades. La mayoría de empresas encuestadas (un 73\%) son micro empresas. La pequeña empresa está presente en un 17\% de los casos y la mediana empresa en un 10\% de los casos. 
Un 64\% de las micro empresas encuestadas pertenece al sector servicios. Del total de empresas, un 23\% exporta sus productos o servicios y un 36\% tienen intención de hacerlo. De las empresas dedicadas a la manufactura, solamente un 32\% exporta sus productos. Estas empresas que exportan mencionan que tienen sistemas de gestión tales como ISO 9001:2015, ISO 14001, regulaciones del Ministerio de Salud, BRC y Global G.A.P.

Del total de empresas dedicadas a la manufactura, solamente el $32 \%$ exporta sus productos; sin embargo, casi el 60\% tiene intención de exportar en el futuro. De las empresas de servicios, un 17\% está exportando actualmente y un 22\% piensa exportar en el futuro. Del total de las empresas que tienen intención de exportar, el 82\% son microempresas. Un 55\% de estas empresas sigue algún tipo de Sistema de Gestión de Calidad.

\section{MANUFACTURA, EXPORTACIÓN Y SISTEMAS DE CALIDAD}

De las empresas dedicadas a la manufactura que actualmente están exportando, solamente el 44\% cuenta con un Sistema de Gestión de Calidad. Entre los sistemas mencionados están ISO 9001:2015, BCR y Global G.A.P. De estas empresas, un $56 \%$ son medianas.

Entre las empresas dedicadas a la manufactura que no están exportando actualmente, pero tienen intención de hacerlo, un 56\% no cuenta con ningún sistema de Gestión de Calidad. Un 31\% sigue regulaciones establecidas por el Ministerio de Salud de Costa Rica.

Del total de las empresas que indican tener un Sistema de Control de Calidad y están dedicadas a la manufactura, un 92\% tiene una Política de Calidad. De las empresas que no cuentan con un Sistema de Gestión de Calidad, solamente un 33\% de ellas tiene Política de Calidad.

\section{SERVICIOS Y SISTEMAS DE CALIDAD}

En el sector servicios, el 50\% de los empresarios manifiesta que no sigue ningún Sistema de Control o Gestión de Calidad. Un 17\% sigue regulaciones del Ministerio de Salud de Costa Rica. Un 7\% de las empresas de servicios están regidos por la Norma ISO 9001:2015. Otras normas mencionadas son: ISO/IEC 17025:2005, Global G.A.P. y Rainforest Alliance.

Todas las empresas que indican tener un Sistema de Control de Calidad y están dedicadas a los servicios, tienen una Política de Calidad. De las empresas que no cuentan con un Sistema de Control de Calidad, solamente un 21\% de ellas tiene Política de Calidad.

\section{EXPORTACIONES Y SISTEMA DE CALIDAD}

En la Figura 1 se puede observar la autoevaluación de las empresas en relación con el aspecto de si tienen o no un Sistema de Control de Calidad. Es interesante recalcar que, alrededor de un $65 \%$ de las empresas que exporta manifiesta que cuenta con algún Sistema de Control de Calidad. Este porcentaje disminuye cuando la empresa no exporta pero tiene intención de exportar a futuro. Finalmente, de las empresas que no exportan del todo, menos de un 30\% tiene algún Sistema de Control de Calidad.

\section{MIPYMES Y SISTEMAS DE CALIDAD}

En la Figura 2 se observa que, conforme las empresas van aumentando en número de empleados, aumenta también la proporción de empresas que cuenta con algún tipo de Sistema de Calidad. Lo anterior en respuesta a la pregunta: ¿Su empresa cuenta con un Sistema de Control de Calidad? Por ejemplo, solamente un 38\% de las micro empresas dice tener un Sistema de Control de Calidad. Esa cifra aumenta a un 62\% en las empresas pequeñas y a un $88 \%$ en las empresas medianas. 
FIGURA 1

CONDICIÓN DE EXPORTACIÓN Y SISTEMA DE CALIDAD

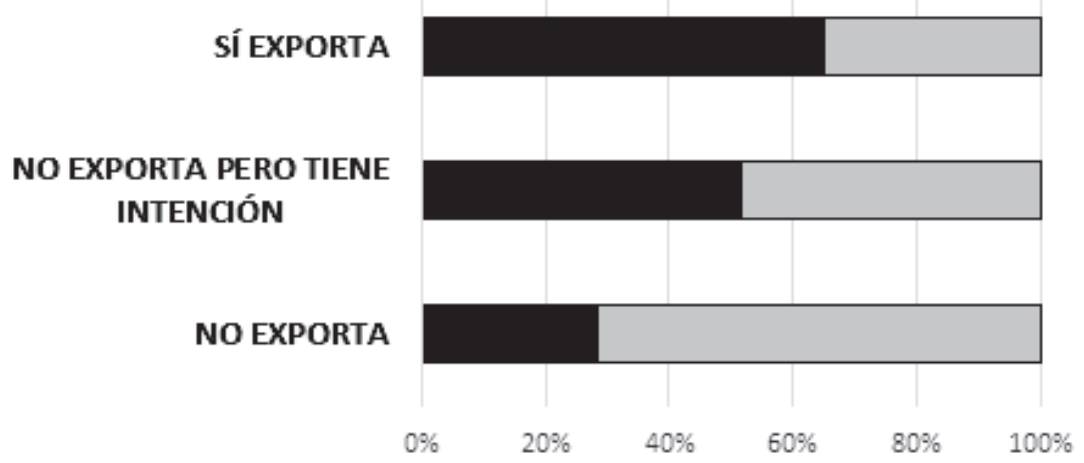

SÍ TIENE UN SISTEMA DE CALIDAD $\square$ NOTIENE UN SISTEMA DE CALIDAD

Fuente: Elaboración propia

FIGURA 2

TAMAÑO DE EMPRESA Y SISTEMA DE CONTROL DE CALIDAD

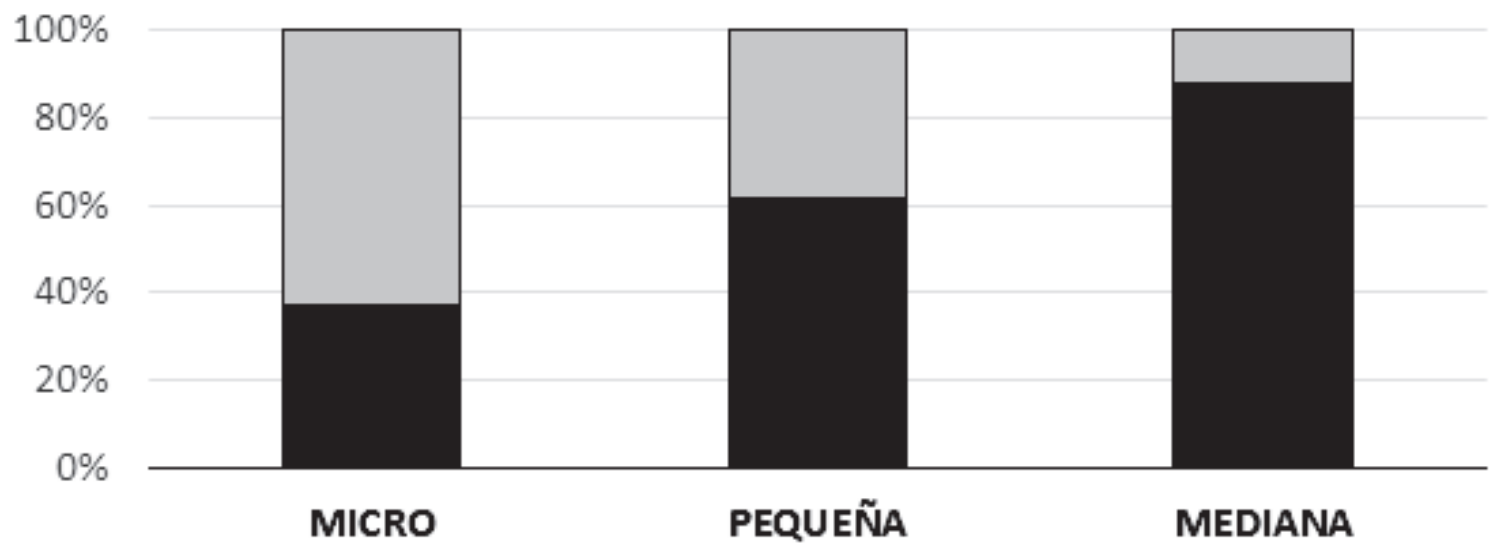

- SÍ TIENE UN SISTEMA DE CALIDAD $\square$ NOTIENE UN SISTEMA DE CALIDAD

Fuente: Elaboración propia

En la Figura 3 se observa un resumen de las respuestas de la escala de Likert del total de las empresas según la sección de la norma ISO 9001:2015 consultada. Se eliminan de este gráfico las respuestas "No aplica". Se muestra en promedio el número de empresas que contestaron en desacuerdo y poco de acuerdo (se catalogan en desacuerdo), y las empresas que contestaron de acuerdo y muy de acuerdo (se catalogan de acuerdo).
La figura 3 permite observar que es en las primeras tres Secciones de la Norma (Contexto de la Organización, Liderazgo y Planificación), en donde los empresarios cumplen más satisfactoriamente los aspectos consultados, según su propia autoevaluación. En la sección Contexto de la Organización, los empresarios respondieron preguntas relacionadas con el nivel de conocimiento y satisfacción de las necesidades de 
FIGURA 3

PORCENTAJE PROMEDIO DE EMPRESAS QUE ESTÁN DE ACUERDO EN CUMPLIR LA CORRESPONDIENTE SECCIÓN DE LA NORMA

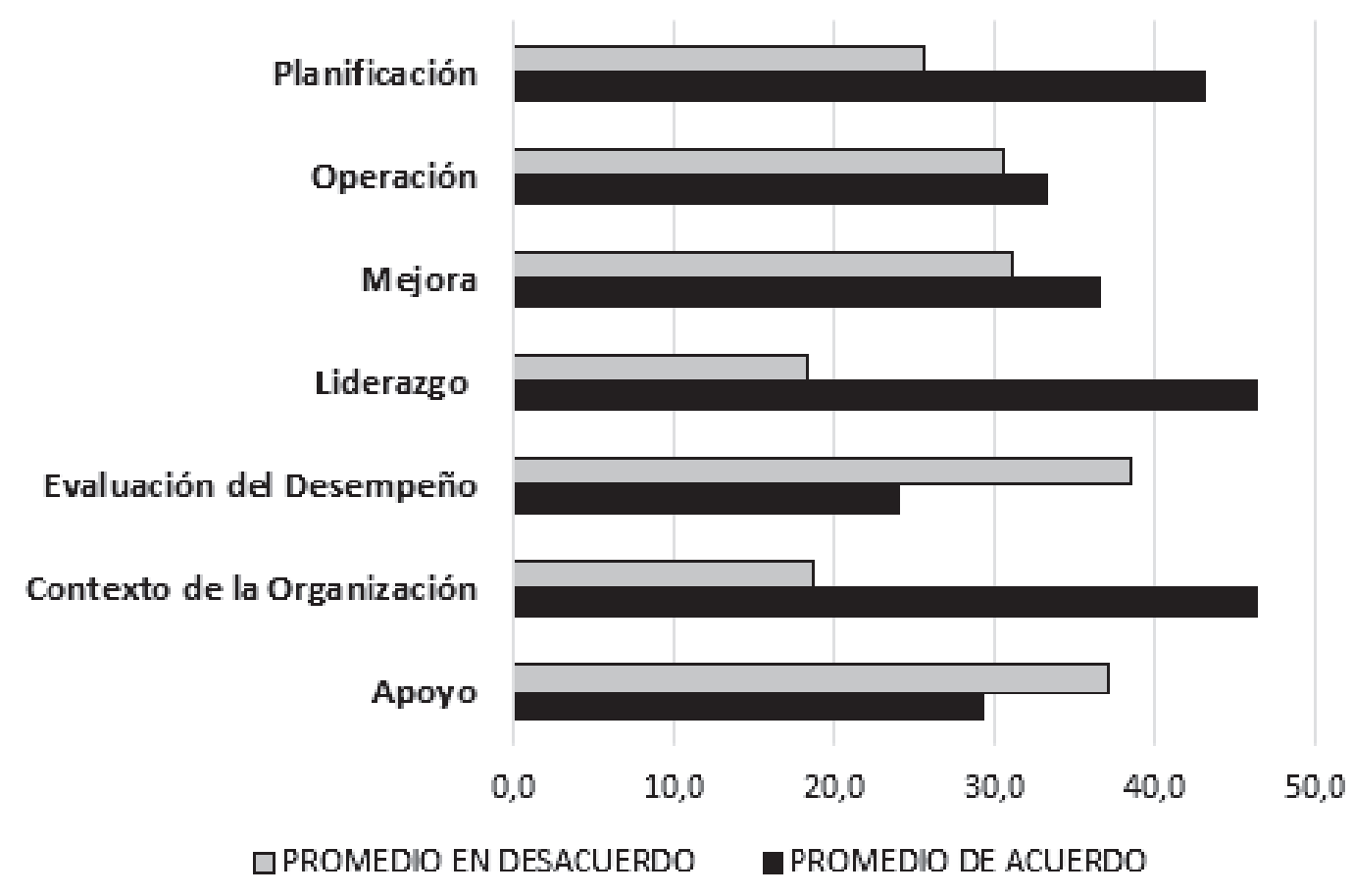

Fuente: Elaboración propia

los clientes y la documentación de sus procesos y operaciones.

A pesar de que esta es una de las secciones mejor calificadas en este estudio, el porcentaje de respuestas "de acuerdo" por parte de los empresarios no alcanza el 50\%. En la sección Liderazgo, se les consulta sobre la enunciación y comunicación de una Política de Calidad y sobre los esfuerzos que hace la empresa por aumentar el nivel de satisfacción de sus clientes. En la sección Planificación, se consulta sobre la existencia y seguimiento de objetivos de calidad y sobre la percepción de los empleados sobre el compromiso de la empresa en temas de calidad.
Una de las secciones de la Norma peor calificada es la de Operación, donde solamente un 33.3\% de las empresas encuestadas está de acuerdo en llevar los controles correspondientes a sus procesos operativos. Esto concuerda con la realidad en la dificultad de implementar los aspectos contemplados en esta sección de la norma, ya que son actividades que generalmente requieren de conocimientos técnicos. La sección peor calificada es Evaluación del Desempeño. Esta incluye el tema de las auditorías internas y mecanismos de seguimiento al grado de satisfacción del cliente. Generalmente, también está asociada con la necesidad de contar con personal capacitado, lo cual es una dificultad para las MIPYMES. 


\section{NIVEL DE CUMPLIMIENTO DE LA NORMA, SE- GÚN TAMAÑO DE EMPRESA}

La Figura 4 muestra el promedio del porcentaje obtenido de las respuestas de acuerdo y muy de acuerdo, según sección consultada de la Norma. Se hace una separación entre micro, pequeñas y medianas empresas para analizar las tendencias. De manera general, las micro empresas tienen una auto-calificación más baja en todas las secciones de la Norma, en comparación con las empresas pequeñas y medianas. Las empresas medianas tienen las auto-calificaciones más altas.

En la sección Contexto de la Organización es donde existe la mayor brecha en las respuestas entre las micro y las pequeñas empresas (41 puntos porcentuales). Es importante recalcar que el 100\% de las empresas pequeñas y medianas considera que conoce y satisface las necesidades de sus clientes, a pesar de que no todas consideran que tienen un sistema de Control de Calidad. El $88 \%$ de las empresas medianas tiene sus procesos debidamente documentados, mientras que solamente el $41 \%$ de las micro empresas cumple con las mismas condiciones.
La sección Liderazgo arroja datos importantes con respecto a la Política de Calidad. En promedio, $67 \%$ de las empresas dice tener una Política de Calidad, pero solamente un 53\% en promedio la comunican a los empleados. Las micro empresas son las que presentan la menor brecha entre tener una Política de Calidad y la comunicación a los empleados. En las secciones Liderazgo y Mejora, prácticamente existe el mismo puntaje entre las empresas pequeñas y medianas, manteniendo una brecha significativa con respecto a las micro empresas.

\section{NIVEL DE CUMPLIMIENTO DE LA NORMA, SE- GÚN SECTOR}

El promedio del porcentaje obtenido de las respuestas "de acuerdo" y "muy de acuerdo", según sección consultada de la Norma, permite analizar si existen diferencias entre las empresas de manufactura y de servicios. En general, el sector manufactura se encuentra levemente por encima del sector servicios, excepto por la sección de la norma correspondiente a planificación. Esto indica que el sector servicios se encuentra mejor

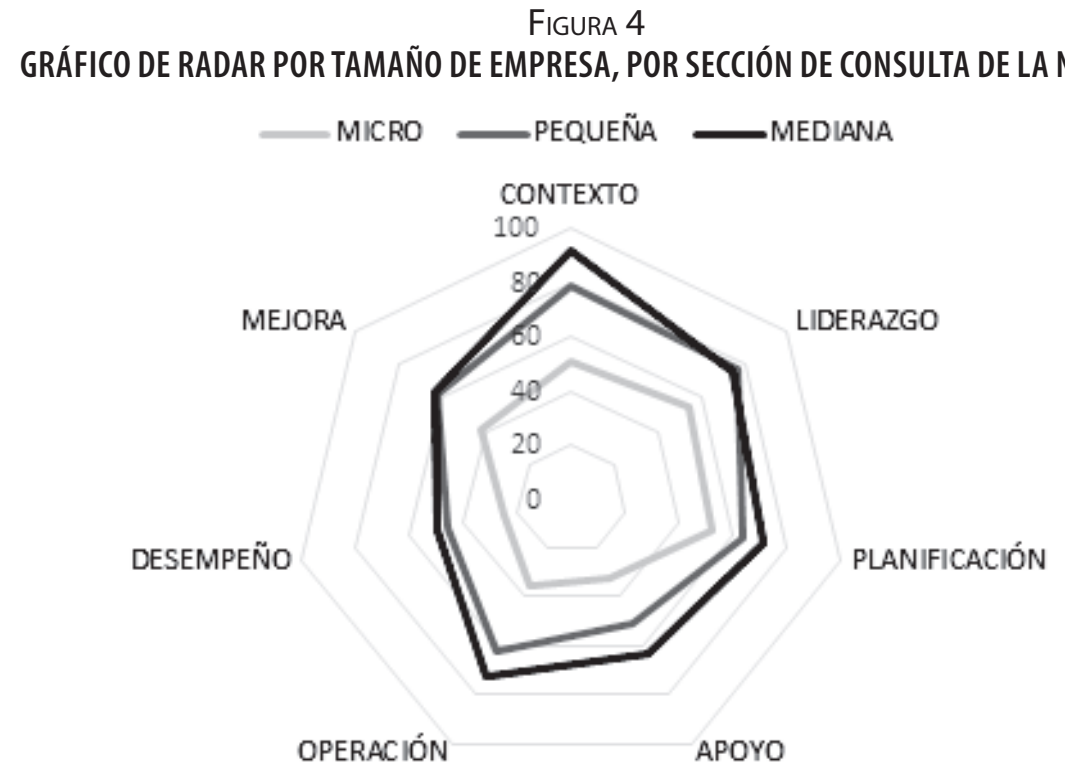

Fuente: Elaboración propia 
auto-calificado, principalmente en lo relativo a la medición y control de los objetivos de calidad. Por otro lado, donde existe mayor diferencia es en las secciones de la norma correspondientes a Operación y Mejora, en donde el sector manufactura está por encima del sector servicios en 7 puntos porcentuales para cada una de las secciones antes mencionadas.

En la Sección de Operación, una de las respuestas más débiles se obtiene del sector servicios, donde solamente un 33\% de las empresas está de acuerdo o muy de acuerdo en tener un sistema de control para evitar que el producto o servicio no conforme le llegue al cliente. En esta respuesta hay una diferencia de 14 puntos porcentuales a favor del sector manufactura.

En la sección de Mejora, las empresas de servicios se catalogan especialmente débiles en el rubro de documentación de no conformidades, ya que solamente un 35\% de ellas está de acuerdo o muy de acuerdo en conservar toda la documentación relativa a las no conformidades. La sección de la Norma peor evaluada por las empresas, es la sección de Evaluación del Desempeño. Con respecto a la pregunta: ¿La empresa realiza auditorías internas de manera periódica para verificar que se cumpla lo establecido en su Sistema de Control de Calidad?, solamente un 37\% de las empresas del sector manufactura está de acuerdo o muy de acuerdo. Apenas un 25\% de las empresas de servicios tiene las mismas características.

Con respecto a la pregunta ¿La empresa cuenta con un mecanismo eficaz para darle seguimiento al grado de satisfacción del cliente?, un 33\% de las empresas del sector manufactura está de acuerdo y muy de acuerdo, y un 35\% del sector servicios tiene las mismas características.

A pesar de los datos anteriores, un poco más de la mitad de las empresas responde positivamente a su situación relativa a la sección Liderazgo. En esta sección, una de las preguntas más importantes es si la empresa cuenta con una Política de Calidad. Las respuestas de ambos sectores son muy similares, obteniendo en promedio un 57.5\% que responde que está de acuerdo o muy de acuerdo con la pregunta anterior.

Otra de las preguntas en la sección Liderazgo es si la Política de Calidad es comunicada a todo el personal. Ambos sectores contestaron estar de acuerdo o muy de acuerdo en un 50\% cada uno. En esta misma Sección de la Norma, la pregunta ¿Constantemente se hacen esfuerzos por aumentar el nivel de satisfacción de sus clientes?, fue contestada de la siguiente manera: un 83\% de las empresas del sector manufactura considera que está de acuerdo o muy de acuerdo con esta pregunta, mientras que un $68 \%$ del sector servicios tiene las mismas percepciones.

Con respecto al Contexto de la Organización (sección 4 de la Norma), las empresas de los sectores manufactura y ambas que respondieron de acuerdo o muy de acuerdo corresponden a un $51 \%$ del total, mientras que las del sector servicios que respondieron de la misma manera representan el $43 \%$.

La Sección peor evaluada de la Norma, es la que se refiere a Evaluación del Desempeño, donde, en promedio, apenas un 23\% de las micro empresas dice tener auditorías internas. En las empresas medianas este porcentaje aumenta a un 50\%. También las empresas suelen tener problemas en consolidar mecanismos eficaces para dar seguimiento al grado de satisfacción del cliente. Apenas un 27\% de las microempresas manifiesta poder contar con un mecanismo para tal fin.

En la sección de Apoyo se encuentra que existen problemas en las empresas con respecto a la formación y experiencia requerida para el personal que trabaja directamente en todo lo relacionado al Sistema de Control de Calidad. En promedio, apenas un 43\% está de acuerdo o muy de acuerdo en que el personal es el adecuado para cumplir ese tipo de funciones. 


\section{NIVEL DE CUMPLIMIENTO DE LA NORMA, SEGÚN SITUACIÓN DE EXPORTACIÓN}

Se estudian por separado las empresas que sí exportan actualmente, las que no exportan y las que tienen intención de exportar en el futuro. No se percibe diferencia en las secciones de la Norma, excepto para las de Contexto y Apoyo, en donde se obtiene en promedio diferencias de hasta 16 puntos porcentuales en las empresas que sí exportan actualmente.

Independientemente de su condición de exportación, las empresas están trabajando de manera muy similar; sin embargo, las que sí exportan están mejor calificadas en los siguientes aspectos: formación y experiencia adecuada del personal que trabaja directamente en Control de Calidad, documentación del Sistema y comunicación del mismo a los empleados, conocimiento y satisfacción de las necesidades de sus clientes y documentación de sus procesos. El rubro mejor calificado por las empresas que sí exportan es el conocimiento y la satisfacción de sus clientes.

\section{NIVEL DE CUMPLIMIENTO DE LA NORMA, SEGÚN SISTEMA DE CALIDAD}

La Figura 5 muestra el promedio del porcentaje obtenido de las respuestas de acuerdo y muy de acuerdo, según sección consultada de la Norma. Se hace una separación entre las empresas que dicen tener un Sistema de Control de Calidad versus las que dicen no tenerlo, con el fin de analizar las tendencias. De manera general, las empresas que dicen tener un Sistema de Control de Calidad tienen una auto-calificación más alta en todas las secciones de la Norma, excepto en la Sección de Evaluación del Desempeño, donde ambos grupos prácticamente empatan en las calificaciones.

Es importante señalar que las empresas que indican tener un Sistema de Control de Calidad, tienen una auto-calificación alta en las primeras secciones de la Norma, es decir, aquellas secciones que NO se refieren a procesos de ejecución. Conforme se avanza en el proceso de ejecución de la Norma, las auto-calificaciones empiezan a bajar, como se observa en la Figura 5.

NIVEL DE CUMPLIMIENTO DE LA NORMA

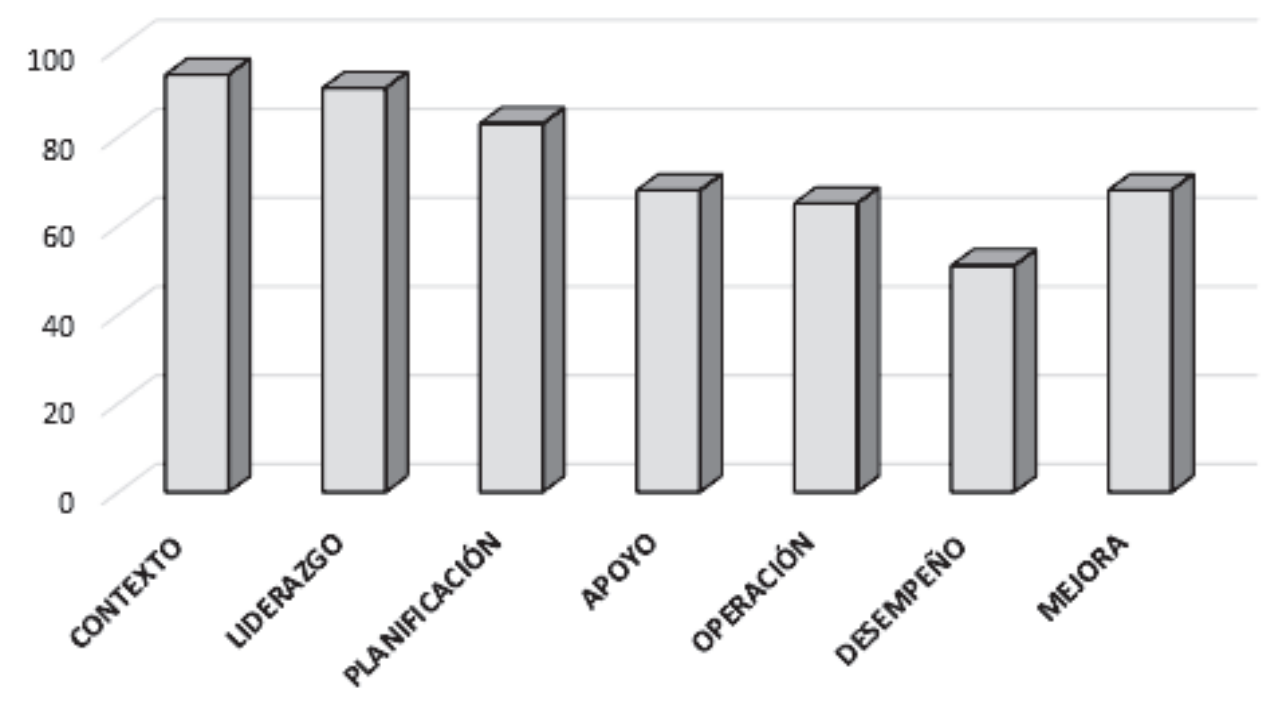

Fuente: Elaboración propia 
Según los resultados de la auto-evaluación, los microempresarios que dicen tener algún tipo de Sistema de Calidad creen conocer y satisfacer las necesidades de los clientes. En la Sección de Planificación, un 92\% de las empresas que dicen tener un Sistema de Control de Calidad, asegura tener definidos objetivos para cumplir con la calidad de sus productos o servicios.

Ante la pregunta abierta: En términos de calidad ¿cuál es el principal problema a resolver en su empresa?, los microempresarios revelaron diferentes áreas de mejora (algunos microempresarios mencionaron más de un área de mejora). Las respuestas que se dieron con mayor frecuencia fueron el control de calidad (10\%), la capacitación de los empleados (8.8\%) y el sistema de gestión de calidad (8.8\%). Entre otros aspectos mencionados están los procedimientos, la comunicación, el seguimiento a los clientes y el servicio post venta.

\section{CONCLUSIONES}

Este estudio permitió comprender cómo se gestiona la calidad en un grupo de MIPYMES costarricenses, considerando variables como cantidad de empleados, la condición de exportación y el sector al que pertenece la empresa. Se desarrolló y aplicó satisfactoriamente un instrumento de evaluación que permitió generar una comparación entre el grado de cumplimiento de los principales elementos de un sistema de gestión y las variables estudiadas.

Con la información de la muestra de MIPYMES analizada, se puede concluir que la cantidad de empleados, la condición de exportación y el sector al que pertenece la empresa influyen en diferente grado en el nivel de atención y compromiso hacia la calidad. El factor que incide de manera más significativa es la cantidad de empleados que tenga la empresa; es decir, a mayor cantidad de empleados, la empresa abarca una más cantidad de los elementos de la norma.
Cuanto más grande sea la empresa en número de empleados, mayor es la probabilidad de que las empresas cuenten con algún tipo de Sistema de Control Calidad, según su propia percepción. Un 38\% de las micro empresas dice tener algún tipo de Sistema de Control de Calidad, mientras que la misma aseveración la hace un $88 \%$ de las empresas medianas. Esta diferencia también se percibe cuando se analizan las diferentes secciones de la norma. Las micro empresas probablemente no cuentan con los recursos ni la cantidad de personal requeridos para la implementación de normas.

La cantidad de empresas que dice tener un Sistema de Control de Calidad, de acuerdo a su propia percepción también aumenta según su condición de exportación. Esta proporción pasa de un 28.6\% para las empresas que no exportan, a un 65\% para las empresas que sí exportan sus productos o servicios. Sin embargo, cuando se analizan las autoevaluaciones por sección de la Norma, no hay diferencias significativas. Esto se debe probablemente a que un 35\% de las empresas que exportan, no practican ni están certificadas en ningún Sistema de Gestión de Calidad, sus clientes no les exigen tales condiciones para comprar sus productos. Por otro lado, las que sí están certificadas no cumplen integralmente los principales elementos de la Norma (en su mayoría están certificadas en ISO 9001:2015 o regulaciones del Ministerio de Salud de Costa Rica).

A nivel general, las empresas de manufactura se auto-califican de mejor manera en prácticamente todas las áreas consultadas de la Norma con respecto a las empresas dedicadas a los servicios. Esto se da con la excepción del área de Planificación, donde la mayor diferencia se percibe en la pregunta sobre objetivos medibles y controlables. A pesar de que los procesos de Gestión de Calidad son especialmente difíciles de implementar en las empresas de servicios debido a que los estándares de desempeño, las necesidades y nivel de satisfacción del cliente no son fáciles de medir, no se detec ta 
diferencia significativa con respecto a las empresas de manufactura de bienes.

En promedio, la sección de la Norma mejor evaluada es Contexto de la Organización, y se da entre las empresas medianas. La sección peor evaluada de la Norma es Evaluación del Desempeño y se da entre las micro empresas.

Las empresas que indican tener un Sistema de Control de Calidad tienen una auto-calificación alta en las primeras secciones de la Norma; es decir, aquellas secciones que $\mathrm{NO}$ se refieren a procesos de ejecución y seguimiento. Existe una tendencia a considerar que se conocen y satisfacen las necesidades de los clientes, pero cuando se evalúa el esfuerzo por conocer el grado de satisfacción del cliente, se obtienen calificaciones más bajas.

En general, las MIPYMES encuestadas tienen mayores dificultades en las áreas de Apoyo, Operación, Evaluación del Desempeño y Mejora, áreas todas relacionadas con procesos de ejecución y seguimiento (Hacer, Verificar y Actuar en el ciclo PHVA). Todos los requerimientos anotados en estas secciones necesitan de personal capacitado para su cumplimiento y seguimiento, ya que se refieren a actividades que demandan conocimiento técnico por parte de su personal (auditorías internas, control de procesos, etc.). Esto coincide con las respuestas de los empresarios en relación con uno de sus mayores problemas en términos de calidad, ya que por un lado les es difícil capacitar a su personal y por otro, les es muy costoso contratar personal capacitado.

\section{REFERENCIAS}

Arce, J. A., Méndez, A., y Villegas, E. (2017). Desarrollo de un Sistema Integrado de Gestión para Micro, Pequeñas y Medianas empresas a partir de la Norma INTE 01-01-09:2013. TEC Empresarial, 11(2) 17-26.
Cámara de Exportadores de Costa Rica. (CADEXCO). (2017). Exportaciones de Mypymes. Recuperado de www.cadexco.org

Chacón, K. (2016, 06 de noviembre). El paso a paso para exportar servicios digitales. El Financiero. Recuperado de https://www.elfinancierocr.com/ tecnologia/el-paso-a-paso-para-exportar-servicios-digitales/DISAWJLPFJGXFDRKDTMYHM37SE/story/

Córdoba, O., y Henderson, A. (2017). La gestión de calidad y el desempeño exportador de las pequeñas y medianas empresas de la Región Huetar Atlántica de Costa Rica. Instituto Tecnológico de Costa Rica. Recuperado de https://repositoriotec.tec. ac.cr/bitstream/handle/2238/9981/gestion_calidad_desempe\%C3\%B1 o_exportador_peque\%C3\%B1as.pdf?sequence $=1$ \&isAllowed $=y$

Instituto Nacional de Aprendizaje. (INA). (2018). Conozca el Tamaño de su empresa. Costa Rica. Recuperado de http://www.pyme.go.cr/cuadro5. php?id=1

ISO 9001. (2015). Sistema de Gestión de Calidad. Recuperado de https://www.iso.org/iso-9001-quality-management.html.

ISO 31000. (2015). Sistema de Gestión de Riesgos. Recuperado de https://www.iso.org/ iso-31000-risk-management.html

Israr, M., y Gangele, A. (2014). A comparative analysis between Small and Medium Scale Manufacturing Company through Total Quality Management Techniques. International Conference on Industrial Engineering and Operations Management Bali, Indonesia.

Ministerio de Economía, Industria y Comercio. (MEIC). (2017). Estado de Situación de las Pyme en Costa Rica. Recuperado de http://reventazon.meic.go. cr/informacion/pyme/2017/informe.pdf.

Ministerio de Economía, Industria y Comercio. (MEIC). (s.f.). Recuperado de http://www.pyme.go.cr/ cuadro5. php?id=1

Rodríguez, A., Bañuelos, K., Rubio, B., y Chávez, J. (2010). Factores internos que afectan la competi- 
tividad de las empresas de la zona Sur de Tamaulipas. Revista Ingeniería Industrial, 4(2), 134-146.

Rubio, A. y Aragón, A. (2009). Recursos Críticos y estrategia en la pyme industrial. ICE Tribuna de Economía, (846), 193-212.

Universidad Nacional Autónoma de México. UNAM. (2018). Congreso Internacional de Contaduría, Administración e Informática. México. Recuperado de http://congreso.investiga.fca.unam.mx

Universidad Cooperativa de Colombia. (UCC). (2018). Sistema de Gestión de Calidad. Recuperado de https://www.ucc.edu.co/sistema-gestion-integral/ Paginas/sistema-gestion-calidad.aspx

Recibido: 25 de febrero de 2019 Aceptado: 23 de mayo de 2019 